\title{
Increased incidence of invasive meningococcal disease of serogroup C / clonal complex 11, Tuscany, Italy, 2015 to 2016
}

P Stefanelli ${ }^{1}$, A Miglietta $^{2}$, P Pezzotti ${ }^{1}$, C Fazio $^{1}$, A Neri ${ }^{1}$, P Vacca $^{1}$, F Voller ${ }^{3}$, FP D'Ancona 45 , R Guerra ${ }^{5}$, S lannazzo $^{5}$, MG Pompa $^{5}$, G Rezza $^{1}$

1. Department of Infectious, Parasitic \& Immuno-mediated Diseases, Istituto Superiore di Sanità, Rome, Italy

2. Epidemiology and Preventive Medicine Unit, Central Tuscany Health Authority, Florence, Italy

3. Health Agency of Tuscany, Florence, Italy

4. National Center for Epidemiology, Surveillance and Health Promotion - Istituto Superiore di Sanità, Rome, Italy

5. Ministry of Health, Directorate-General of health prevention, Rome, Italy

Correspondence: Paola Stefanelli (paola.stefanelli@iss.it)

Citation style for this article:

Stefanelli P, Miglietta A, Pezzotti P, Fazio C, Neri A, Vacca P, Voller F, D’Ancona FP, Guerra R, lannazzo S, Pompa MG, Rezza G. Increased incidence of invasive meningococcal disease of serogroup C / clonal complex 11, Tuscany, Italy, 2015 to 2016. Euro Surveill. 2016;21(12):pii=30176. DOI: http://dx.doi.org/10.2807/156o7917.ES.2016.21.12.30176

Article submitted on o9 March 2016 / accepted on 24 March 2016 / published on 24 March 2016

We report an increase of serogroup C Neisseria meningitidis invasive meningococcal disease in Tuscany. From January 2015 to end February 2016, 43 cases were reported, among which 10 were fatal, compared to two cases caused by serogroup C recorded in 2014 and three in 2013. No secondary cases occurred. Thirty-five strains belonged to $\mathrm{C}: \mathrm{P}_{1} .5-1,10-8: \mathrm{F}_{3}-$ 6:ST-11(cC11). Control measures have been adopted and immunisation campaigns implemented. Studies on risk factors and carriage are ongoing.

In this report we present an unexpected increase of invasive meningococcal disease (IMD) in Tuscany, Italy, since January 2015, leading to a total of 43 cases, of whom 10 were fatal, due to infection with serogroup C Neisseria meningitidis. In Italy, serogroup $C$ is the second most common serogroup (31\% of the 115 cases with a known serogroup in 2014), after serogroup B ( $48 \%$ of the 115 cases in 2014) [1]. In Tuscany, the total number of IMD cases was 16 in 2014 and 12 in 2013, with two and three cases caused by serogroup C, respectively [1].

\section{Epidemiological features}

From January 2015 to February 2016, 43 laboratoryconfirmed cases of IMD due to serogroup C N. meningitidis (31 in 2015, 12 in 2016) were reported from the Regional Health Authority of Tuscany (RHAT) to the Italian National Surveillance System for Invasive Bacterial Disease (IBD). No secondary cases were detected. The incidence rate (IR) of serogroup $C$ cases was higher compared with the previous years: 0.83 per 100,000 inhabitants in 2015 and 1.98 in the first two months of 2016 whereas the average IR for 2012-2014 was 0.08 per 100,000 inhabitants ranging from 0.05 in 2014 to 0.11 in 2012.
The National Reference Laboratory at the National Institute of Health (Istituto Superiore di Sanità, ISS) received 22 bacterial isolates and 18 clinical samples (10 from cerebrospinal fluid (CSF) and 8 from blood) from 40 patients; for three cases material was not available. Thirty-five out of the 40 samples analysed were confirmed as C:P1.5-1,10-8:F3-6:ST-11 (cc11).

The median age of the 43 cases reported in the period was 28 years (range: $9-82$ ), with the age group 20-29 years being the most affected ( $n=15 ;$ IR: 3.9/100,000), followed by the age group $9-19$ years $(n=10 ; I R$ : 2.6/100,000). Interestingly, 18 cases were reported among people over 30 years old (IR: 0.5/100,000), and 11 among people over 55 years old (IR: 0.6/100,000). There was no notable difference between males and females, with 21 cases registered among women and 22 among men.

The main clinical manifestations were: sepsis only $(n=18)$, sepsis and meningitis $(n=14)$, followed by meningitis only $(n=11)$. Ten patients aged between 12 and 82 years died.

Information on vaccination status was available for 42 of the 43 cases detected between January 2015 and February 2016. Five patients had been vaccinated with meningococcal $C$ conjugate (MCC) vaccine. Apart from one case in an individual aged 62 years, vaccinated on the day of the symptom onset, in the remaining four cases aged 9, 12, 17, 22 years, the vaccine was administered in 2006, 2007, 2013 and 2008, respectively. In the latter cases, the apparent vaccine failure was likely to be due to the relatively short duration of the protection induced by one vaccine dose [2]. Two of the 
Annual distribution of serogroup C invasive meningococcal disease cases by outcome, January 2000-February 2016 ( $\mathrm{n}=111$ cases) (A) and monthly distribution, January 2015- February 2016 (B) ( $n=43$ cases), Tuscany, Italy

A.

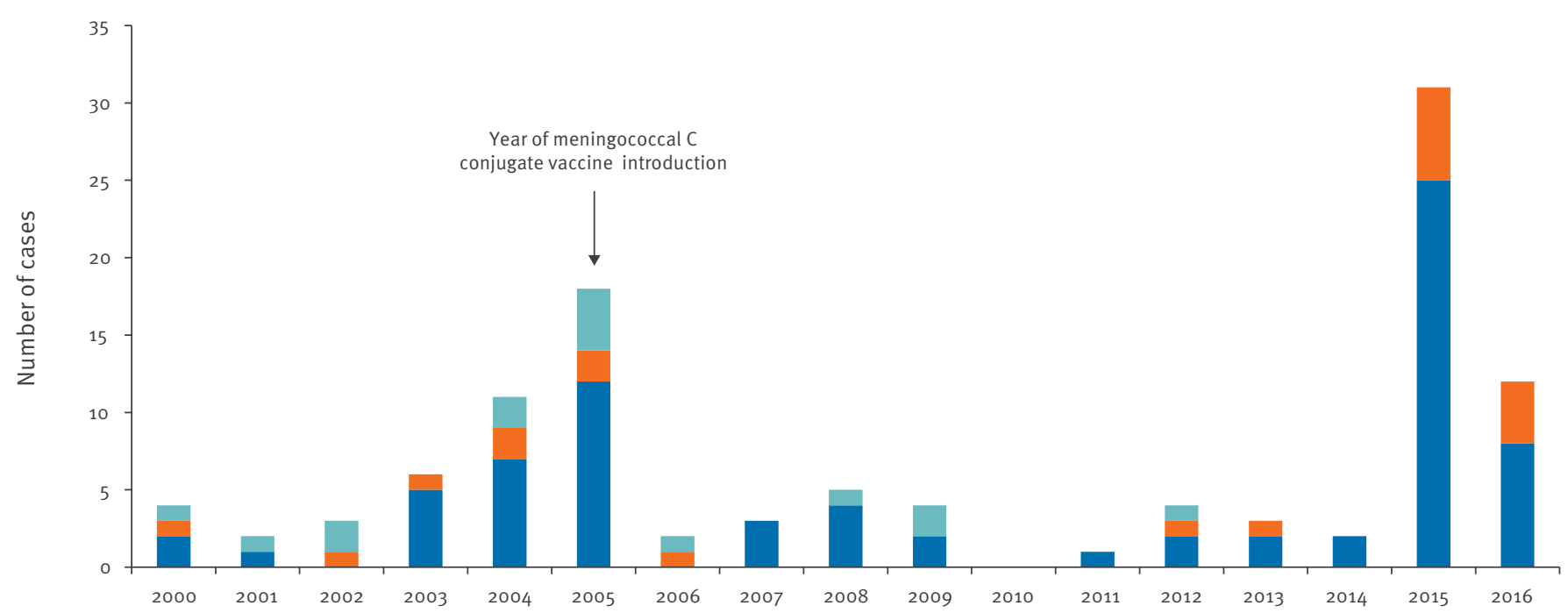

Year

B.

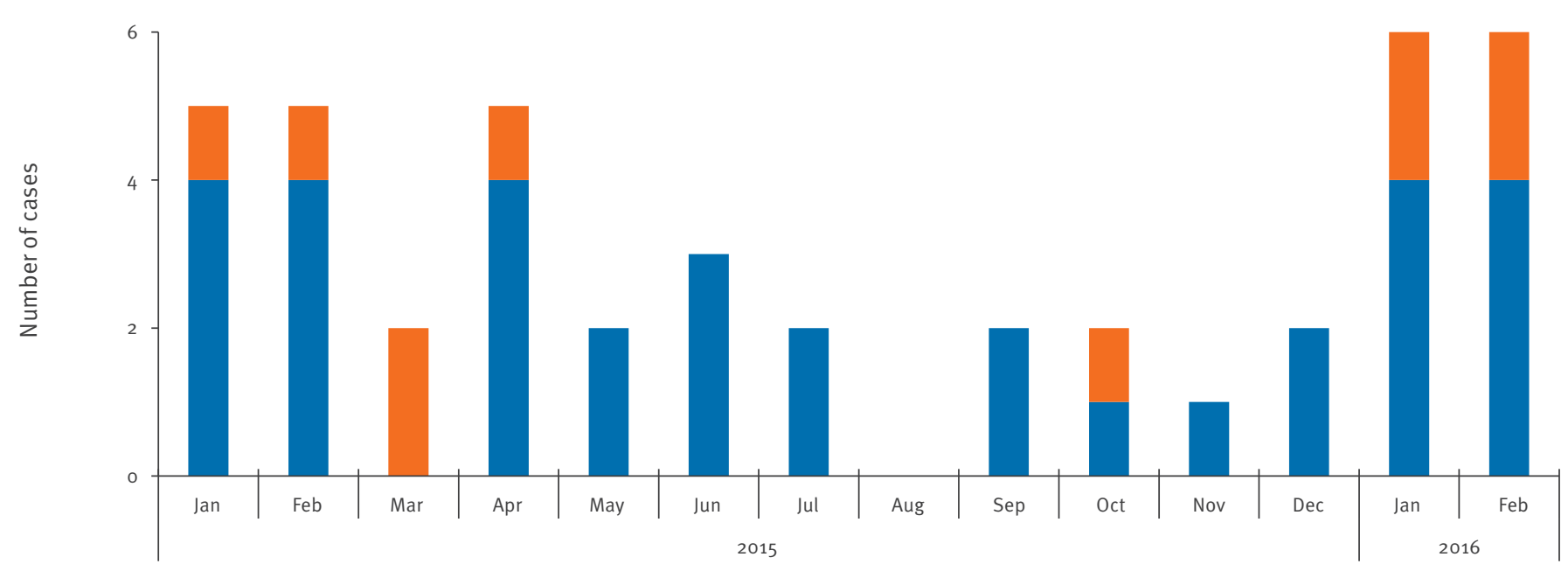

Year

Cases Fatal cases No information available

vaccinated cases developed meningitis and two sepsis (one of them died).

Figure 1 shows the case distribution by year from January 2000 to February 2016 and by month for January 2015 to February 2016, and Figure 2 presents the geographical distribution of the cases. An increase in the number of cases was observed since January 2015 in a densely populated area in the north of Tuscany, between the cities of Florence, Prato, and Empoli. Between January and April 2015, the cases were confined in this area. At the end of the spring, some cases occurred in the coastal area of Tuscany, between Pisa and Viareggio, an area frequented by young people during the summer. From the end of September 2015, the cases reappeared in the original area, where 12 cases with four deaths occurred in the first two months of 2016. 


\section{FIGURE 2}

Number (A) and incidence rate per 100,000 inhabitants (B) of serogroup C invasive meningococcal disease cases, by municipality of symptom onset; Tuscany, Italy, January 2015 to February 2016

A.

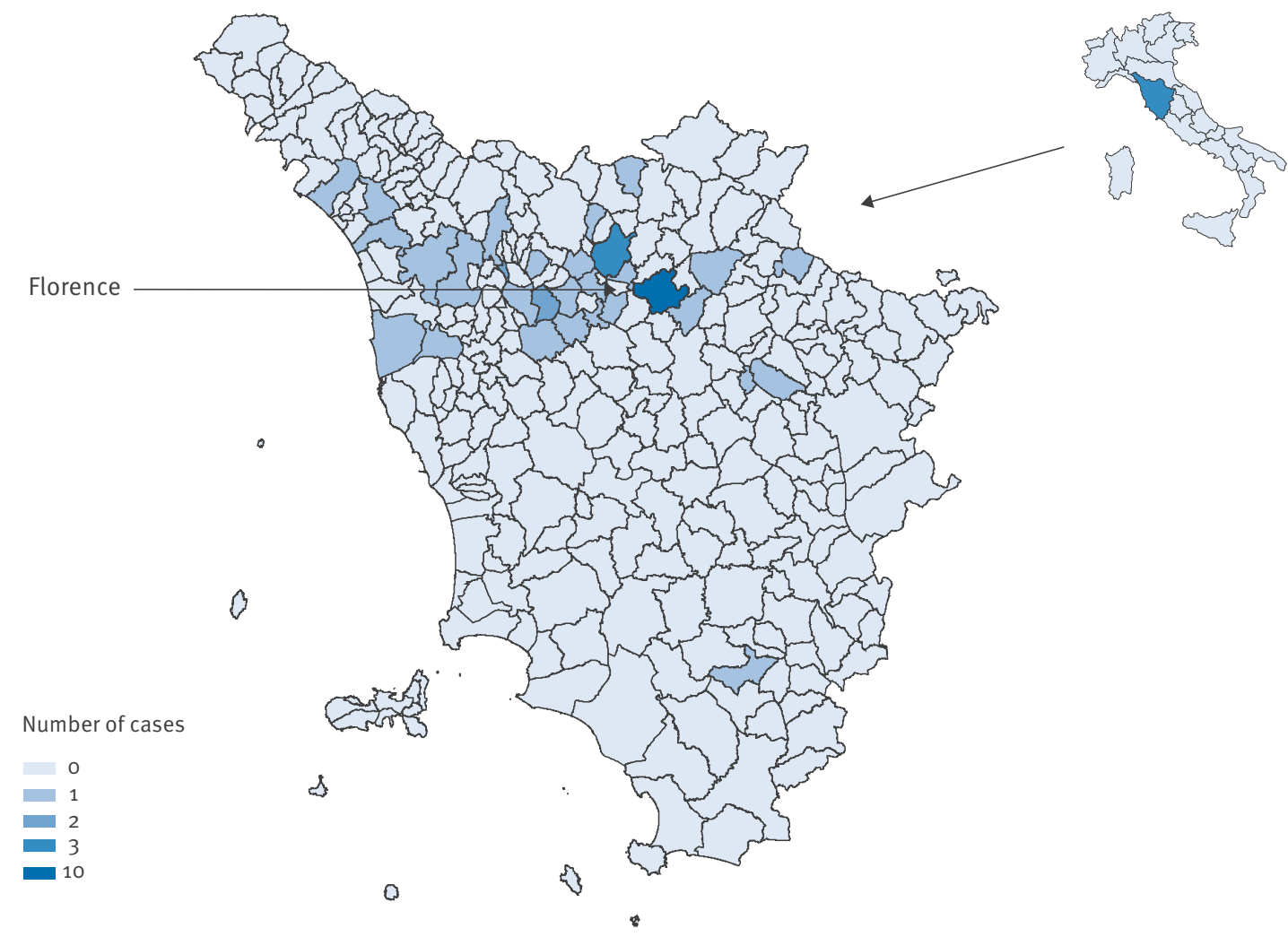

B.

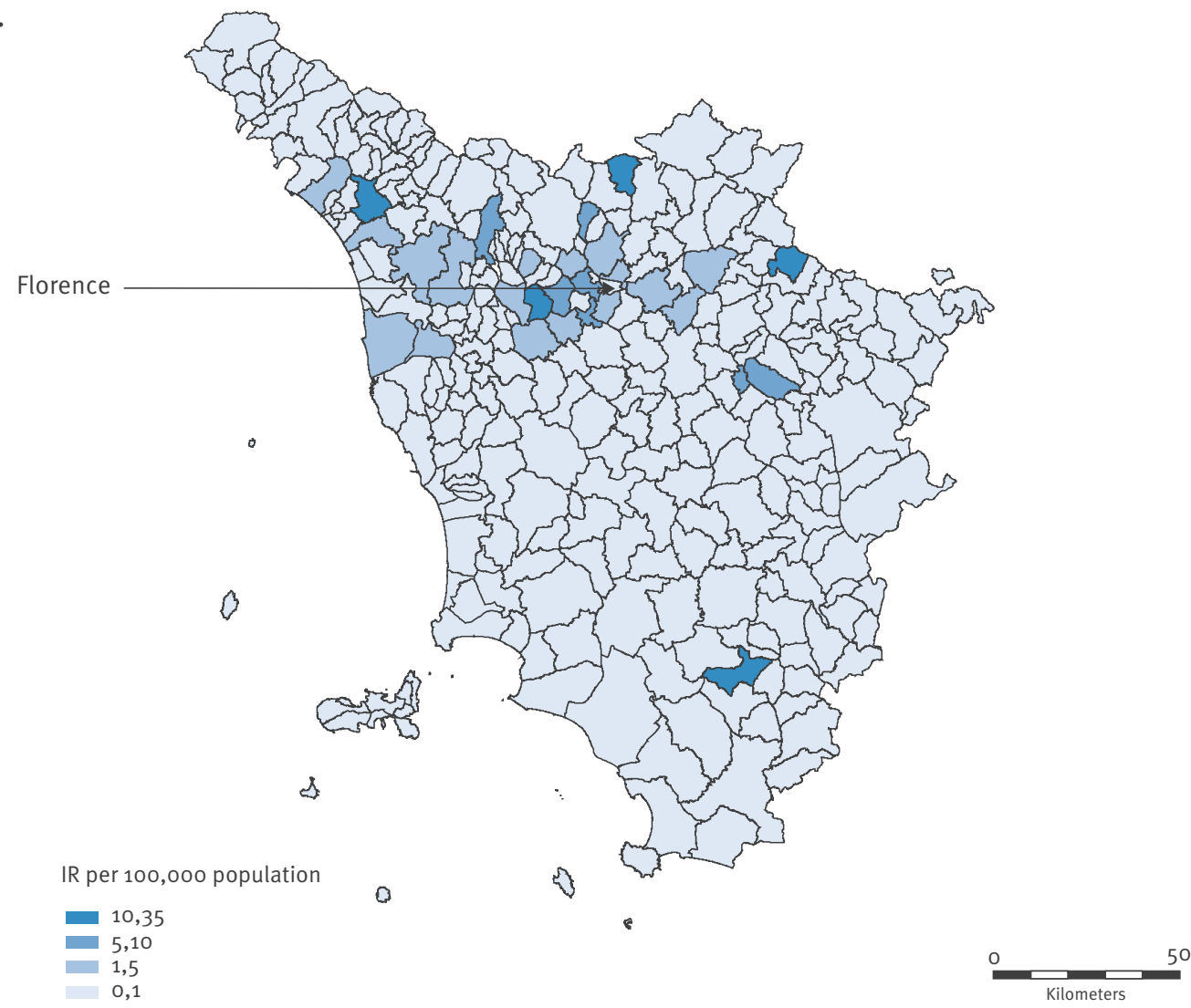

The incidence rate was calculated as the ratio between the number of cases in the study period (14 months) divided by the person-years of exposure (calculated as the people living in each municipality of Tuscany in 2015 (www.demo.istat.it), multiplied by the exposure time in years (i.e. 14 months $/ 12=1.17$ years)). 


\section{Background}

Meningococcal serogroup C strains cc11, are known to cause invasive disease burden worldwide [3] and are responsible for high mortality rates among cases [4]. Outbreaks due to $\mathrm{C}: \mathrm{P}_{1} .5-1,10-8: \mathrm{F}_{3}-6: \mathrm{ST}-11$ (cC11) of IMD were reported in Germany in 2013 [5], in France in 2014 [6], in Italy among staff members of a cruise ship at the port of Livorno, Tuscany, in 2012 [7], and in two clusters in northern Italy, in December 2007 and July 2008 , respectively, showing a high rate of septicaemia and fatal outcome [8].

The RHAT introduced the MCC vaccine in the regional immunisation schedule in 2005 , with three doses to all children at three, five, and 13 months of age (subsequently turning to a single dose at 13 months, in 2008), and a catch-up immunisation until six years of age with a single dose. In 2007, the RHAT also implemented a catch-up vaccination programme with a single dose of MCC targeting the age group 11 to 14 year-olds. At national level, MCC was introduced in the Italian National Immunisation Plan in 2012 [9].

\section{Public health response}

Since March 2015, the RHAT involved ISS in the microbiological characterisation (including genomic analysis) and public health response. The factors contributing to an excess of IMD cases due to such hyper-virulent meningococcal $C$ strain remain currently unknown but investigations are ongoing. An immunisation campaign has been implemented and continuously adapted to the evolution of the epidemiological situation. Starting with 30 March 2015, a single dose of meningococcal (ACYW) polysaccharide-protein conjugate vaccine has been actively offered free-of-charge to the age group 11-19 years-old, even if already vaccinated with MCC in childhood (letters with invitations have been sent to individuals within this age group); the vaccine has been offered to individuals aged $20-44$ years residing in the area of the local health units that reported at least one case of serogroup C N. meningitidis since 2015 (Arezzo, Empoli, Florence, Lucca, Massa, Pistoia, Pisa, Prato and Versilia). Up to 31 December 2015, 120,272 children and teenagers aged between 11 and 19 years were vaccinated, leading to a coverage of $42.5 \%$ in this age group; 109,101 individuals aged between 20 and 44 years were vaccinated (vaccine coverage 14\%). On 16 February 2016, due to the increasing number of cases in age groups not previously included in the vaccination target groups, the immunisation campaign was extended to the whole Tuscany Region and to older people, using the monovalent vaccine as an alternative option to the tetravalent vaccine, maintaining the active offer only to 11-20 years age group.

Following the advice of the European Centre for Disease Prevention and Control (ECDC), standard operating procedures for public health management of IMD, including contact tracing and administration of chemoprophylaxis to close contacts, already established before 2015, were extended and included the recommendation to offer vaccination to unimmunised people [10].

\section{Conclusion}

In order to investigate the reasons of this unusual increase in the number of cases, to assess possible epidemiological links between cases, and to identify specific groups of population at risk for both meningococcal serogroup C disease and carriage status, research protocols consisting in detailed investigation of the cases and $N$. meningitidis cross sectional carriage surveys are going to be implemented in Tuscany, shortly. Molecular characterisation of meningococcal of serogroup $\mathrm{C}$ isolates, in particular those belonging to the finetype $\mathrm{C}: \mathrm{P}_{1} .5-1,10-8: \mathrm{F}_{3}-6: \mathrm{ST}-11$ (cC11), is in progress, to define the correlation with isolates reported in other countries $[5,6]$. The same analysis is now performed also in IMD cases occurring in other Italian Regions, in order to verify the spread of the strain involved in the outbreak in Tuscany to other Italian areas.

These investigations will help to better understand the dynamic of the ongoing circulation of this hyper-virulent meningococcal serogroup $C$ strain and to identify groups of population at higher risk, in order to address specific prevention strategies, develop preparedness plans for an effective response to future IMD threats, and to address the ongoing public health concern.

At present, the Italian Health Authorities have enhanced IMD surveillance activities but did not consider necessary to provide special recommendations for people travelling to Tuscany.

\section{Acknowledgements}

This work was funded by the Italian Ministry of Health-CCM Project 'Sorveglianza delle malattie invasive da Neisseria meningitidis, Streptococcus pneumoniae, Haemophilus influenzae' 2014. These data were made available via the Neisseria Multi Locus Sequence Typing website (http:// pubmlst.org/neisseria) developed by Keith Jolley and sited at the University of Oxford.

\section{Conflict of interest}

None declared.

\section{Authors' contributions}

PS proposed the study and together with AM, PP and GR conceived the study design. PS drafted the manuscript. AM and PP performed the statistical analysis of IMD clinical cases and together with GR further drafted the text. FV contributed to the public health responses regarding the IMD cases at regional level. CF, AN, PV performed the microbiological analyses on samples under the supervision of PS. FPD, SI, RG contributed to the revision of the analysis of IMD cases and together with RG and MGP further revised the manuscript. GR critically revised the manuscript. All authors have read and approved the submitted manuscript. 


\section{References}

1. Italian National Health Institute. (Istituto Superiore di Sanità, ISS). Dati di sorveglianza delle malattie batteriche invasive aggiornati al 23 dicembre 2015. [Surveillance data on invasive bacterial diseases updated on 23 December 2015]. Italian. Available from: http://www.iss.it/binary/mabi/cont/ Report_MBI_20151223_v4.pdf

2. Ishola DA, Borrow R, Findlow H, Findlow J, Trotter C, Ramsay $M E$. Prevalence of serum bactericidal antibody to serogroup C Neisseria meningitidis in England a decade after vaccine introduction.Clin Vaccine Immunol. 2012;19(8):1126-30. DOI: 10.1128/CVI.05655-11 PMID: 22647271

3. Watkins ER, Maiden MC. Persistence of hyperinvasive meningococcal strain types during global spread as recorded in the PubMLST database.PLoS One. 2012;7(9):e45349. DOI: 10.1371/journal.pone.0045349 PMID: 23028953

4. Smith I, Caugant DA, Høiby EA, Wentzel-Larsen T, Halstensen A. High case-fatality rates of meningococcal disease in Western Norway caused by serogroup C strains belonging to both sequence type (ST)-32 and ST-11 complexes, 19852002. Epidemiol Infect. 2006;134(6):1195-202. DOI: 10.1017/ So950268806006248 PMID: 16650328

5. Marcus U, Vogel U, Schubert A, Claus H, Baetzing-Feigenbaum J, Hellenbrand W, et al. A cluster of invasive meningococcal disease in young men who have sex with men in Berlin, October 2012 to May 2013. Euro Surveill. 2013:18(28):20523. DOI: 10.2807/1560-7917.ES2013.18.28.20523 PMID: 23870095

6. Aubert L, Taha M, Boo N, Le Strat Y, Deghmane AE, Sanna A, et al. Serogroup $C$ invasive meningococcal disease among men who have sex with men and in gay-oriented social venues in the Paris region: July 2013 to December 2014. Euro Surveill. 2015;20(3):21016. DOI: 10.2807/1560-7917.ES2015.20.3.21016 PMID: 25635319

7. Stefanelli P, Fazio C, Neri A, Isola P, Sani S, Marelli P, et al. Cluster of invasive Neisseria meningitidis infections on a cruise ship, Italy, October 2012. Euro Surveill. 2012;17(50):20336. PMID: 23241233

8. Stefanelli P, Fazio C, Sofia T, Neri A, Mastrantonio P. Serogroup $C$ meningococci in Italy in the era of conjugate menC vaccination.BMC Infect Dis. 2009;9(1):135. DOI: 10.1186/14712334-9-135 PMID: 19698137

9. Italian Ministry of Health. Piano Nazionale. Prevenzione Vaccinale. (PNPV) 2012 - 2014. [National Vaccination Prevention Plan 2012-2014]. Italian Available from: http:// www.salute.gov.it/imgs/c_17_pubblicazioni_1721_allegato.pdf

10. European Centre for Disease Prevention and Control (ECDC). Public health management of sporadic cases of invasive meningococcal disease and their contacts. Stockholm: ECDC; 2010. Available from: http://ecdc.europa.eu/en/publications/ Publications/1010_GUI_Meningococcal_guidance.pdf

\section{License and copyright}

This is an open-access article distributed under the terms of the Creative Commons Attribution (CC BY 4.0) Licence. You may share and adapt the material, but must give appropriate credit to the source, provide a link to the licence, and indicate if changes were made.

This article is copyright of the authors, 2016. 